\title{
High expression of TOP2A in hepatocellular carcinoma is associated with disease progression and poor prognosis
}

\author{
HONGYU CAI ${ }^{1,2}$, BINGFENG SHAO ${ }^{1}$, YUAN ZHOU ${ }^{1}$ and ZHONG CHEN $^{2,3}$ \\ ${ }^{1}$ Department of Hepatobiliary Surgery, The Affiliated Tumor Hospital of Nantong University, Nantong, Jiangsu 226361; \\ ${ }^{2}$ Department of General Surgery, The First Affiliated Hospital of Soochow University, Soochow, Jiangsu 215006; \\ ${ }^{3}$ Department of Hepatobiliary Surgery, The Affiliated Hospital of Nantong University, Nantong, Jiangsu 226001, P.R. China
}

Received July 28, 2019; Accepted July 9, 2020

DOI: $10.3892 / 01.2020 .12095$

\begin{abstract}
Hepatocellular carcinoma (HCC) is a common malignant tumor in the clinic. Although there are increasing numbers of available treatment methods, their therapeutic effects are not satisfactory. The clinical indicators commonly used to predict the prognosis of HCC include tumor size, degree of cirrhosis, degree of tumor differentiation and tumor microvascular invasion; however, there are currently no molecular indicators that can predict the prognosis of HCC. Due to the differences in the progression of liver cancer among individuals, there is a growing need for prognostic biomarkers to accurately stratify patients for appropriate risk-adaptive treatment. The DNA topoisomerase 2- $\alpha$ (TOP2A) gene, which is located on human chromosome 17, encodes DNA topoisomerase II $\alpha$. Previous studies have demonstrated that TOP2A indicates a poor prognosis in patients with various types of tumors, but no such studies are currently available on HCC. By analyzing the differential expression of TOP2A in 50 pairs of tumor and paracancerous tissue samples in The Cancer Genome Atlas (TCGA) database, the present study revealed that the expression of TOP2A was significantly higher in tumor tissue compared with that in paracancerous tissue $\left(\mathrm{P}=6.319 \times 10^{-16}\right)$. In the collected clinical samples, the mRNA expression levels of TOP2A were significantly upregulated in $\mathrm{HCC}$ tumor tissues compared with those in the paracancerous tissues $\left(\mathrm{P}=6.40 \times 10^{-3}\right)$, suggesting that TOP2A was associated with the occurrence and development of liver cancer. In addition, the associations between TOP2A expression, clinicopathological features and prognosis were analyzed using a multi-center large sample dataset from TCGA database, and the results demonstrated that high expression of TOP2A was associated with a higher $\mathrm{T}$ stage, poorer clinical stage and
\end{abstract}

Correspondence to: Dr Zhong Chen, Department of Hepatobiliary Surgery, The Affiliated Hospital of Nantong University, 20 Xisi Road, Nantong, Jiangsu 226001, P.R. China

E-mail:drchenz@hotmail.com

Key words: DNA topoisomerase 2- $\alpha$, hepatocellular carcinoma, prognosis higher histological grade compared with those in patients with low TOP2A expression. High expression of TOP2A was also identified to be associated with a poor prognosis of HCC, particularly in Asian populations. These results suggested that high expression of TOP2A in HCC tissues may be closely associated with tumor progression and metastasis, which may be used as a biological indicator to predict tumor prognosis in clinical practice.

\section{Introduction}

According to the WHO data released in May 2019, the number of new liver cancer cases was 841080 worldwide in 2018 ranked sixth, and the number of deaths was 781631, the death rate ranked third among all known types of cancer. In Asia, liver cancer ranks fourth in morbidity and third in mortality (1). The main treatment methods of primary liver cancer include surgical resection, local ablation, transhepatic arterial chemotherapy and embolization (TACE), molecular targeting and immunotherapy under exploration (2). However, the treatment outcomes remain still unsatisfactory. As an important indicator for the diagnosis of hepatocellular carcinoma (HCC), compared to healthy subjects, the serum $\alpha$-fetoprotein (AFP) is increased to varying degrees in $\leq 70 \%$ of HCC cases. However, it has been demonstrated that AFP levels do not predict the tumor stage or prognosis (3). The clinical indicators commonly used to predict the prognosis of HCC include tumor size, degree of cirrhosis, tumor differentiation and microvascular invasion $(4,5)$. Due to the differences in the progression of liver cancer among individuals, there is a growing need for prognostic biomarkers to accurately stratify patients for appropriate risk-adaptive treatment.

DNA topoisomerase is an enzyme that catalyzes the transformation of DNA topologies; it is present in the nucleus of living organisms and not only serves a catalytic role in the breaking and binding of DNA strands, but also affects the three-dimensional structure of DNA (6). DNA topoisomerase type I (TOP1) breaks the single strand, whereas DNA topoisomerase II (TOP2) cuts off the double chain (7). Although the two TOPs are highly homologous in structure, their distribution in the organism is significantly different, and they are not produced by the same transportation process (8). TOP2 is divided into two subtypes, of which the 1,531 amino acid 
$170 \mathrm{kDa}$ subtype encoded by the TOP2A gene is topoisomerase II $\alpha$, and the 1,621 amino acid $180 \mathrm{kDa}$ subtype encoded by the TOP2B gene is topoisomerase II $\beta$ (9). TOP2A serves an important role in DNA synthesis and transcription, as well as chromosome segregation during mitosis (10). The location of the human TOP2A gene is on chromosome 17, and its expression is associated with cell proliferation and the cell cycle; its production and degradation are regulated by the cell cycle, and it decreases rapidly after completing mitosis $(11,12)$. Previous studies have reported that the accumulation of the TOP2A protein occurs due to the stable upregulation of TOP2A transcription $(13,14)$.

In addition to its physiological function, TOP2A has been reported to be a sensitive and specific marker of active proliferating cells in the late $S, G$ and $M$ phases of the cell cycle, indicating its importance in cancer research (15). Increased cell proliferation is a hallmark of malignant tumors, and the expression of TOP2A in proliferating cells is significantly increased; therefore, its expression levels reflect the tumor proliferation level to a certain extent and indicates invasive behavior and poor prognosis (16). In colorectal (17), liver (18), esophageal (19) and gastric cancer (20), the expression levels of TOP2A are higher compared with those in the adjacent normal tissues. TOP2A can be used as a potential biomarker to predict the prognosis of patients with malignant tumors and screen out high-risk cases in order to enable individualized treatment (21). In a study on triple-negative breast cancer, a significant association was observed between the TOP2A gene and chromosome 17 polysomy (CEP17) status, and TOP2A has a certain predictive effect on the therapeutic effect of anthracycline drugs (22). Wittmann et al (23) confirmed that the high expression of TOP2A was associated with tumor metastasis and death outcome by analyzing 102 cases of nephroblastoma. A large-scale retrospective study has demonstrated that TOP2A high expression is associated with poor differentiation and neural invasion of esophageal cancer, and is also an independent risk factor affecting the prognosis of esophageal cancer (24). De Resende et al (25) have reported that high expression of TOP2A protein is associated with severe lymphatic vascular invasion and a low relapse-free survival rate in prostate cancer. In addition, in breast cancer, TOP2A expression is associated with Ki67 expression, mitotic count, tumor grade and nipple infiltration, and high expression of TOP2A adversely affects prognosis (26). High expression of TOP2A in tumors, such as pancreatic cancer, prostate cancer and breast cancer is also associated with regional lymph node metastasis and metastasis to other organs, and these behaviors lead to a poor prognosis (27-29). In studies on endometrial (30) and adrenal gland (31) cancer, high expression of TOP2A has been demonstrated to indicate a poor prognosis. However, to the best of our knowledge, no studies on TOP2A expression and prognosis in primary liver cancer are currently available.

The present study aimed to investigate the association between TOP2A expression and the clinicopathological characteristics of patients with liver cancer, and to conduct survival analysis on the prognosis of the entire liver cancer cohort and the Asian cohort in The Cancer Genome Atlas (TCGA) database to explore the feasibility of TOP2A as a prognostic indicator.

\section{Materials and methods}

Patients and samples. The study protocol and acquisition of tissue specimens were approved by the Clinical Ethics Committee of The Affiliated Tumor Hospital of Nantong University (approval no. 2018-024). The patients provided informed consent for participation and publication of the present study. The inclusion criteria were patients who were clinically diagnosed with HCC and were about to undergo surgical resection, regardless of tumor size, sex and age. All specimens were verified as HCC by pathological analysis and non-HCC patients will be excluded. A total of $15 \mathrm{HCC}$ and paired paracancerous liver tissue specimens were collected during hepatectomy at The Affiliated Tumor Hospital of Nantong University between March and August 2018. Among the 15 cases, 7 were males and 8 were females, age range, 47-77 years old with a mean age of 63 years. The liver tissues within $2 \mathrm{~cm}$ from the edge of the tumor was obtained as paracancerous tissues. The specimens were immediately stored in liquid nitrogen until further experiments. These same specimens have been used in a previous study (32).

Reverse transcription-quantitative polymerase chain reaction (RT-qPCR)

RNA preparation. Total RNA was extracted from 15 pairs of HCC and paired paracancerous tissues by TRIzol ${ }^{\circledR}$ reagent (Invitrogen; Thermo Fisher Scientific, Inc.).

Synthesis of cDNA chain 1. Total RNA was reverse transcribed into cDNA, and Beacon Designer 8.14 (http://www. premierbiosoft.com/molecular_beacons/index.html) was used to design the RT-PCR primers. Total RNA was extracted from tumor or paracanerous tissue using TRIzol ${ }^{\circledR}$ reagent (Invitrogen; Thermo Fisher Scientific Inc). The RNA was subsequently treated with RNase-free DNase I (Roche Applied Science, Inc). Reverse transcription was performed using the BcaBest ${ }^{\circledR}$ RNA PCR kit (Takara Biotechnology, Co., Ltd) with a temperature protocol of $30^{\circ} \mathrm{C}$ for $5 \mathrm{~min}$, followed by $65^{\circ} \mathrm{C}$ for $30 \mathrm{~min}$ and $98^{\circ} \mathrm{C}$ for $5 \mathrm{~min}$. The quantitative polymerase chain reaction was conducted using the StepOnePlus ${ }^{\circledR}$ Real-Time PCR System (Applied Biosystems; Thermo Fisher Scientific Inc.). The primer sequences were as follows: TOP2A forward, 5'-CATTGAAGACGCTTC GTTATGG-3' and reverse, 5'-CAGAAGAGAGGGCCAGTT GTG-3'; and $\beta$-actin forward, 5'-ATAGCACAGCCTGGA TAGCAACGTAC-3' and reverse, 5'-CACCTTCTACAATGA GCTGCGTGTG-3'. The qPCR mix comprised the following: $10 \mu 1 \mathrm{SYBR}^{\circledR}$ Green I (BioRuler, Inc.), $0.8 \mu 1(10 \mu \mathrm{mol} / \mathrm{l})$ qPCR upstream and downstream primers, $2 \mu 1 \mathrm{cDNA}, 0.4 \mu \mathrm{l}$ 50X ROX reference dye and deionized water to $20 \mu 1$. The reaction conditions were as follows: $95^{\circ} \mathrm{C}$ for $1 \mathrm{~min}$, followed by 40 cycles of $95^{\circ} \mathrm{C}$ for $30 \mathrm{sec}$ and $60^{\circ} \mathrm{C}$ for $40 \mathrm{sec}$. All experiments were repeated three times. The relative quantitative was used by $2^{-\Delta \Delta \mathrm{Cq}}$ method (33). The results were analyzed by paired Student's t-test.

Gene expression data and clinical data from TCGA database. Gene expression and clinical data from patients with HCC were obtained from TCGA-liver hepatocellular carcinoma (LIHC) dataset (https://www.cancer. 
gov/about-nci/organization/ccg/research/structural-genomics/tcga) A total of 371 cases and 424 samples of gene expression data were downloaded. Among them, 50 tumor tissue samples were paired with paracancerous tissues, and the rest of the 374 samples were tumor tissue. A total of 377 cases of clinical data files were obtained, 371 cases retained after matching with tissue specimen data when analyzing the prognostic factors. Among the 371 cases, 252 were from male and 119 were from female patients, with a mean age of 59.44 years (age range, 16-90 years). Among the 371 cases, one case provided 2 tumor tissue specimens, another case provided 3 tumor tissue specimens, and the remaining 369 cases each provided one tumor tissue specimen. The total number of specimens was 374 . Of the 371 cases, there was one case provided 3 tumor samples, and another case provided 2 tumor samples, so the total number of tumor samples was 374. Among these 374 tumor specimens, some clinical information was missing. Seven cases lacked T stage, 17 lacked clinical stage and 2 lacked histological grade. These cases were deleted accordingly during analysis.

Differential TOP2A expression analysis. Perl language (www. perl.org) was used to organize the downloaded data, and $\mathrm{R}$ (version 3.6.0) (34) was used to analyze the differences in TOP2A expression between paracancerous and tumor tissues. 'Limma' (35) and 'beeswarm' (36) packages were used to analyze the differences in the expression of TOP2A between paracancerous and tumor tissues, and Perl was used to pair the tumor tissues with paracancerous tissues for analysis.

Associations between TOP2A expression, clinicopathological characteristics and prognosis. To study the associations between TOP2A expression and pathological grade, clinical and $\mathrm{T}$ stage, logistic regression analysis was performed using R. Univariate and multivariate analyses were conducted for age, sex, pathological grade, clinical staging, $\mathrm{T}$ stage and TOP2A expression levels using the 'survival' package, and Kaplan-Meier analysis was conducted to compare the survival of patients stratified into high and low TOP2A expression groups.

Statistical analysis. The quantitative data are presented as the mean $\pm \mathrm{SD}$, and the qualitative data are presented as counts or percentages. $\mathrm{R}$ (version 3.6.0) was used for statistical analysis. The median TOP2A expression was used as the cut-off value to determine the high and low expression groups. The exact median expression value was assigned to the low expression group. Paired t-test was used to analyze the differences in TOP2A mRNA expression in 15 pairs of fresh tumors and paracancerous tissues. When analyzing TOP2A expression data downloaded from TCGA, all 374 tumor samples were compared with 50 paracancerous tissues using unpaired Student's t-test, and 50 pairs of tumors and paracancerous tissues from the same patients were compared using paired Student's t-test. Kruskal-Wallis tests were performed to determine the associations between TOP2A expression and clinicopathological characteristics. Cox multivariate regression was used to ascertain the expression of TOP $2 \mathrm{~A}$ in tumor and adjacent tissues, and Renyi test was used for survival analysis.

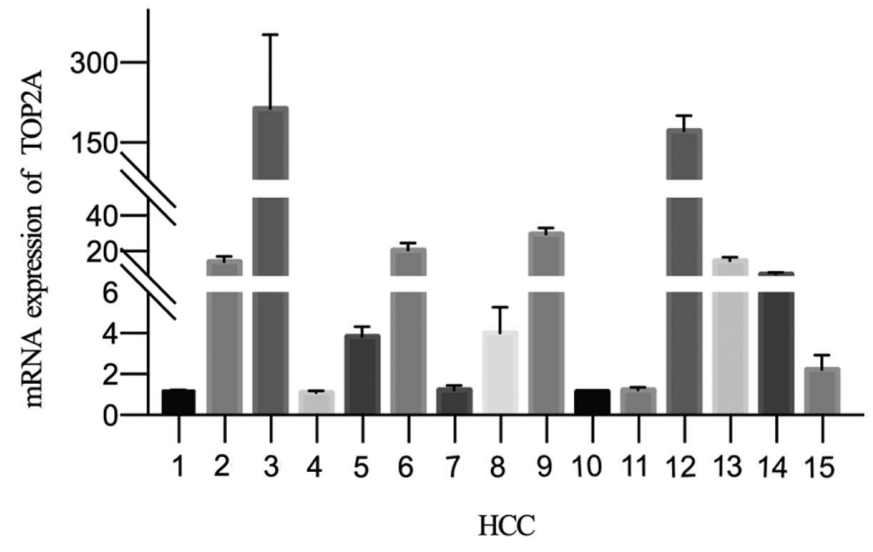

Figure 1. TOP2A expression in HCC and paracancerous fresh tissue samples was determined by reverse transcription-quantitative PCR. The X-axis represents the patient's serial number. HCC, hepatocellular carcinoma; TOP2A, DNA topoisomerase II $\alpha$.

\section{Results}

TOP2A mRNA expression in tumor and paracancerous tissues. To determine the differences in TOP2A expression between tumor and paracancerous tissues, RNA was extracted from 15 pairs of tumor and paracancerous tissues and analyzed by RT-qPCR. The mRNA expression levels of TOP2A in tumor tissues was significantly higher compared with those in paracancerous tissues normalized to $\beta$-actin ( $\mathrm{P}=0.0064$; Fig. 1$)$.

TOP2A expression and clinicopathological features in TCGA-liver hepatocellular carcinoma (LIHC) dataset. In the TCGA-LIHC dataset, the expression of TOP2A in 374 tumor tissue specimens was higher compared with that in 50 paracancerous tissues $\left(\mathrm{P}=1.85 \times 10^{-27}\right.$; Fig. $\left.2 \mathrm{~A}\right)$. In 50 paired samples of tumor and paracancerous tissues, the expression levels of TOP2A in tumor tissues were higher compared with those in the paired paracancerous tissues $\left(\mathrm{P}=6.319 \times 10^{-16}\right.$; Fig. $\left.2 \mathrm{~B}\right)$.

The association between the expression of TOP $2 \mathrm{~A}$ and $\mathrm{T}$ stage, clinical stage and histological grade were analyzed by logistic regression. The expression levels of TOP $2 \mathrm{~A}$ in stages T3-T4 were higher compared with those in stages T1-T2 (OR, 1.86; 95\% CI, 1.16-3.02; $\mathrm{P}=0.011$; Table I). The expression of TOP2A in clinical stage II tumors was higher compared those in with stage I (OR, 1.88; 95\% CI, 1.11-3.17; $\mathrm{P}=0.012)$; the expression of TOP2A in stage III-IV was higher compared with that in stage I $\left(\mathrm{OR}, 2.71 ; 95 \% \mathrm{CI}, 1.59-4.69 ; \mathrm{P}=2.90 \times 10^{-4}\right)$; and the expression of TOP2A in histological grade G3-G4 was higher compared with that in grade G1-G2 (OR, 3.22; 95\% CI, 2.07-5.07; P=3.12×10-7; Table I). Kruskal-Wallis test identified significant differences in the expression of TOP2A among different histological grades, clinical stages and T stages (Fig. 3).

High expression of TOP $2 A$ is associated with a poor prognosis. To determine the association between TOP2A expression and prognosis, Kaplan-Meier analysis was performed in data subsets stratified by clinicopathological factors. As presented in Fig. 4, the overall survival of patients with low expression of TOP2A was higher compared those with high 
Table I. Logistic regression of TOP2A expression and patient clinicopathological characteristics.

\begin{tabular}{lcccr}
\hline Characteristic & $\mathrm{n}$ & Total, $\mathrm{n}$ & Odds ratio (95\% CI) & P-value \\
\hline T stage, T3-T4 vs. T1-T2 & 93 vs. 274 & 367 & $1.86(1.16-3.02)$ & $1.10 \times 10^{-2 a}$ \\
Clinical stage & & 347 & & \\
Stage II vs. stage I & 86 vs. 171 & 257 & $1.88(1.11-3.17)$ & $1.20 \times 10^{-2 a}$ \\
Stage III-IV vs. stage I & 90 vs. 86 & 176 & $2.71(1.59-4.69)$ & $2.90 \times 10^{-4 a}$ \\
Histological grade, G3-G4 vs. G1-G2 & 137 vs. 235 & 372 & $3.22(2.07-5.07)$ & $3.12 \times 10^{-7 a}$ \\
\hline
\end{tabular}

${ }^{a} \mathrm{P}<0.05$. All the patients were from the TCGA cohort. TOP2A, DNA topoisomerase II $\alpha$; TCGA, The Cancer Genome Atlas.
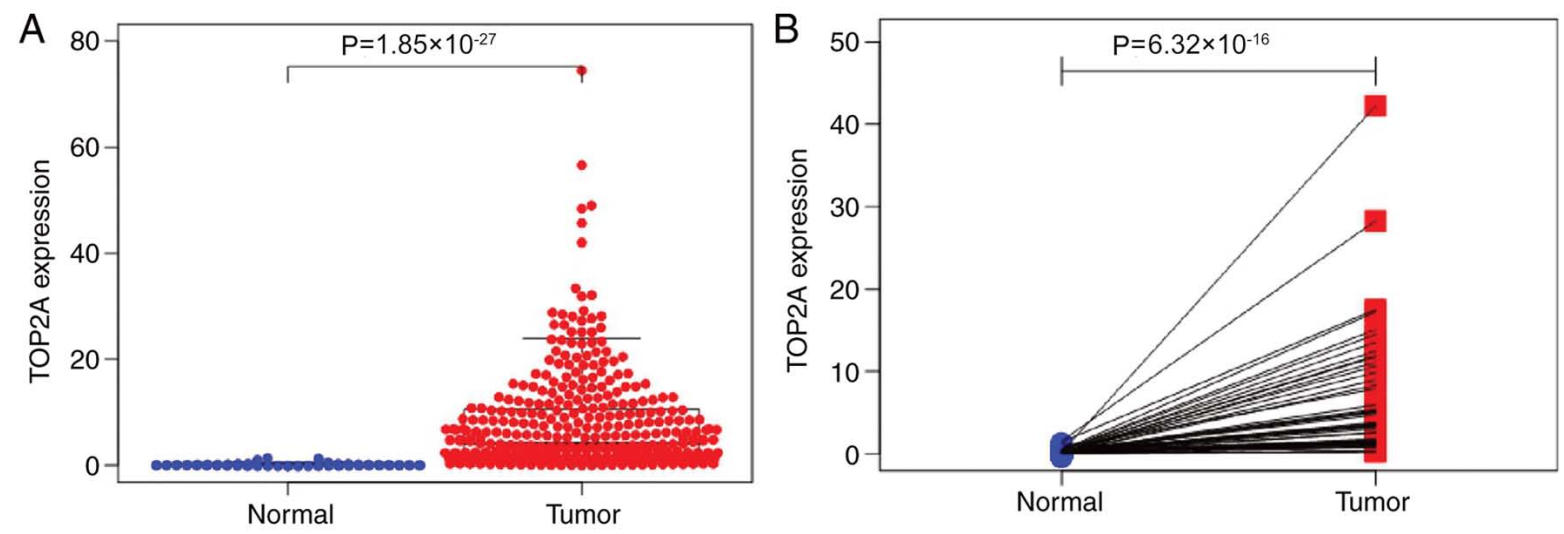

Figure 2. Expression of TOP2A is higher in HCC compared with that in adjacent tissues. (A) TOP2A expression in 50 paracancerous liver tissue samples and 374 HCC samples obtained from TCGA. (B) TOP2A expression in 50 paired tumor and paracancerous tissues obtained from TCGA. HCC, hepatocellular carcinoma; TOP2A, DNA topoisomerase II $\alpha$; TCGA, The Cancer Genome Atlas.

expression $\left(\mathrm{P}=2.20 \times 10^{-4}\right.$; Fig. 4A). The 5-year survival rate of the TOP2A high expression group was $48.7 \%$, whereas that of the low expression group was $55.4 \%$. Among different clinical stages, the overall survival of the high expression group does not present with improved survival in any of the presented groups. Clinical stage I-III $\left(\mathrm{P}=1.90 \times 10^{-4}\right.$; Fig. 4B), stage II-III $\left(\mathrm{P}=5.74 \times 10^{-4}\right.$; Fig. 4D), stage III $\left(\mathrm{P}=5.99 \times 10^{-3}\right.$; Fig. 4F $)$ and stage III-IV ( $\mathrm{P}=2.31 \times 10^{-2}$; Fig. 4G) exhibited significant differences between the two groups. No significant differences were observed between the high and low TOP2A expression groups in the overall survival of patients at stage I and stage II $(\mathrm{P}=0.17$; Fig. $4 \mathrm{C}$ and $\mathrm{P}=0.13$; Fig. $4 \mathrm{E})$, and no independent survival analysis was performed for patients at stage IV as the number of patients was low.

In the multivariate analysis, high expression of TOP2A was the only independent factor for poor prognosis of HCC (HR, 1.240; 95\% CI, 1.071-1.435; P=2.23 $\times 10^{-6}$; Fig. 5A).

Effects of TOP2A expression on survival in different ethnic groups. Kaplan-Meier analysis was performed using data from Asian and non-Asian patients (all ethnic groups) in the dataset to identify whether differences in ethnicity were associated with the effects of TOP2A on patient survival in HCC. As presented in Fig. 6, the overall survival of Asian patients in the high and low TOP2A expression groups was significantly different $\left(\mathrm{P}=5.03 \times 10^{-5}\right)$, whereas among the non-Asian groups, the overall survival differences due to abnormal TOP2A expression were not significant $(\mathrm{P}=0.212)$. Multivariate analysis results indicated that high expression of TOP2A was an independent prognostic factor in the Asian population (HR, 2.005; 95\% CI, 1.493-2.692; $\mathrm{P}=3.74 \times 10^{-6}$; Fig. 5B).

\section{Discussion}

HCC is one of the most common malignant tumors worldwide (37). After years of development, the treatment methods for HCC have gradually evolved from early surgical resection, liver transplantation, TACE, chemotherapy and radiotherapy to molecular targeted therapy combined with immunotherapy (38). AFP was previously recommended by the guidelines as a serum marker for monitoring HCC (39). A study by Agopian et al (40) reported that among 665 patients with HCC, $31.3 \%$ had AFP levels within the normal range. Although AFP is still used in the clinic, it cannot be used to monitor patients who present with normal levels of AFP (41). The European Association for the Study of the Liver (EASL) recommends that in addition to AFP, vascular endothelial growth factor and angiopoietin 2 can also be used as prognostic markers (42). In addition, efforts to identify new effective prognostic biomarkers for HCC are ongoing. According to previous reports, high expression levels of collagen type XXIV alpha 1 chain (43), ubiquilin 2 (44), sushi, von Willebrand factor type A, EGF and pentraxin domain containing 1 (45), acyl-CoA synthetase long chain family member 4 (46) and YTH N6-methyladenosine RNA binding protein 1 (47) are associated with the progression of 

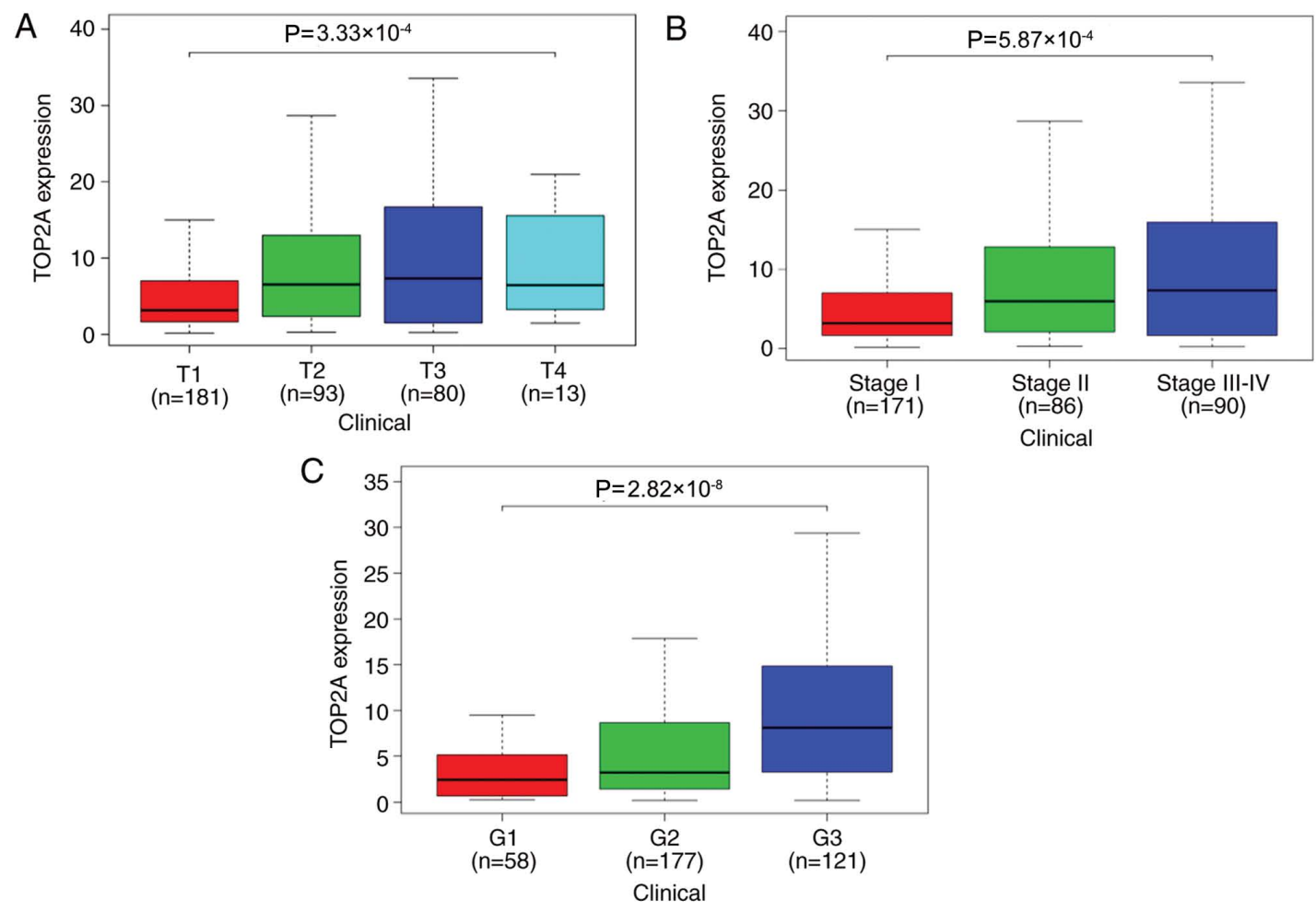

Figure 3. Associations between TOP2A expression and patient clinicopathological characteristics. (A-C) TOP2A expression levels in patients stratified into groups by (A) T stage, (B) clinical stage and (C) histological grade. All samples were obtained from the TCGA. T, tumor; G, tumor grade; TOP2A, DNA topoisomerase II $\alpha$; TCGA, The Cancer Genome Atlas.
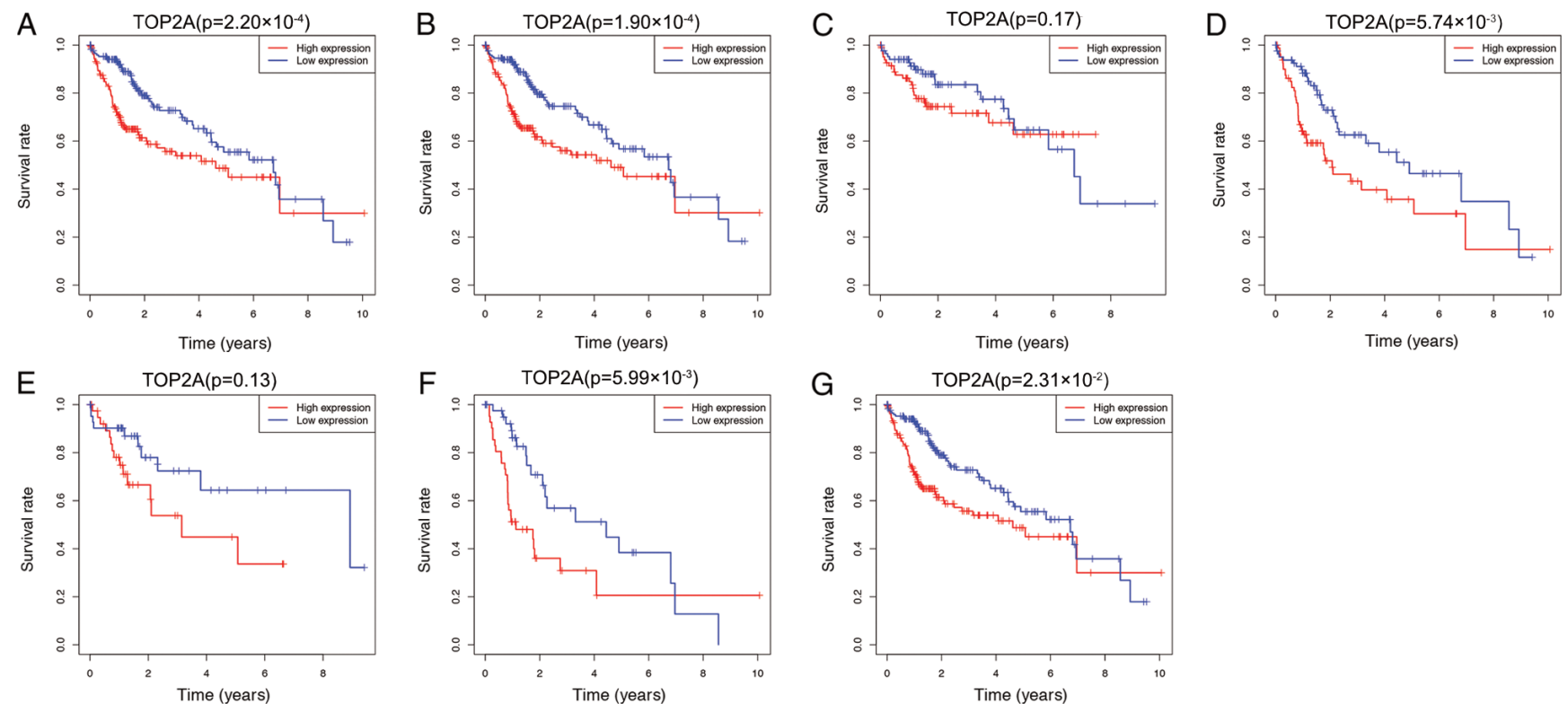

Figure 4. Overall survival of patients with HCC stratified into TOP2A high and low expression groups. (A) All patients. (B-G) Patients with (B) stage I-III, (C) stage I, (D) stage II-III, (E) stage II, (F) stage III and (G) stage III-IV HCC. All samples were obtained from the TCGA. HCC, hepatocellular carcinoma; TOP2A, DNA topoisomerase II $\alpha$; TCGA, The Cancer Genome Atlas.

HCC. Another study has demonstrated that in addition to the American Joint Committee on Cancer staging system, a long non-coding RNA-based risk score is another important factor affecting the overall survival of patients with HCC (48). However, none of the above methods are currently widely used in the clinic. Therefore, there is an urgent need to identify new prognostic markers for HCC.
TOP2A serves an important role in various biological behaviors of cells, including DNA replication, chromosome separation, chromatin concentration and gene expression (49). As a DNA replication- and cell division-regulating enzyme, TOP2A is the main target of several anticancer drugs, such as doxorubicin, etoposide, and mitoxantrone (50). High expression levels of TOP2A in various types of tumors, such as lung 
A

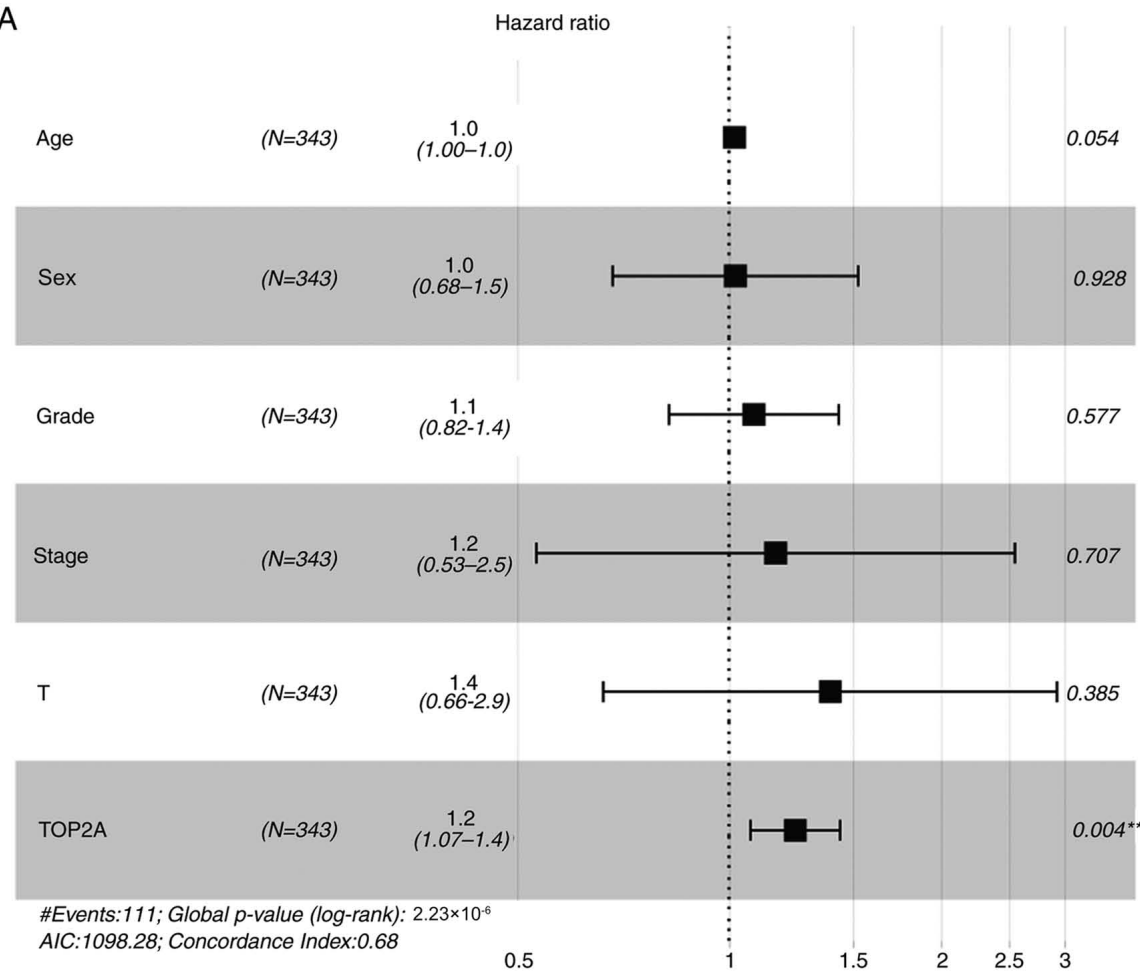

B

Hazard ratio

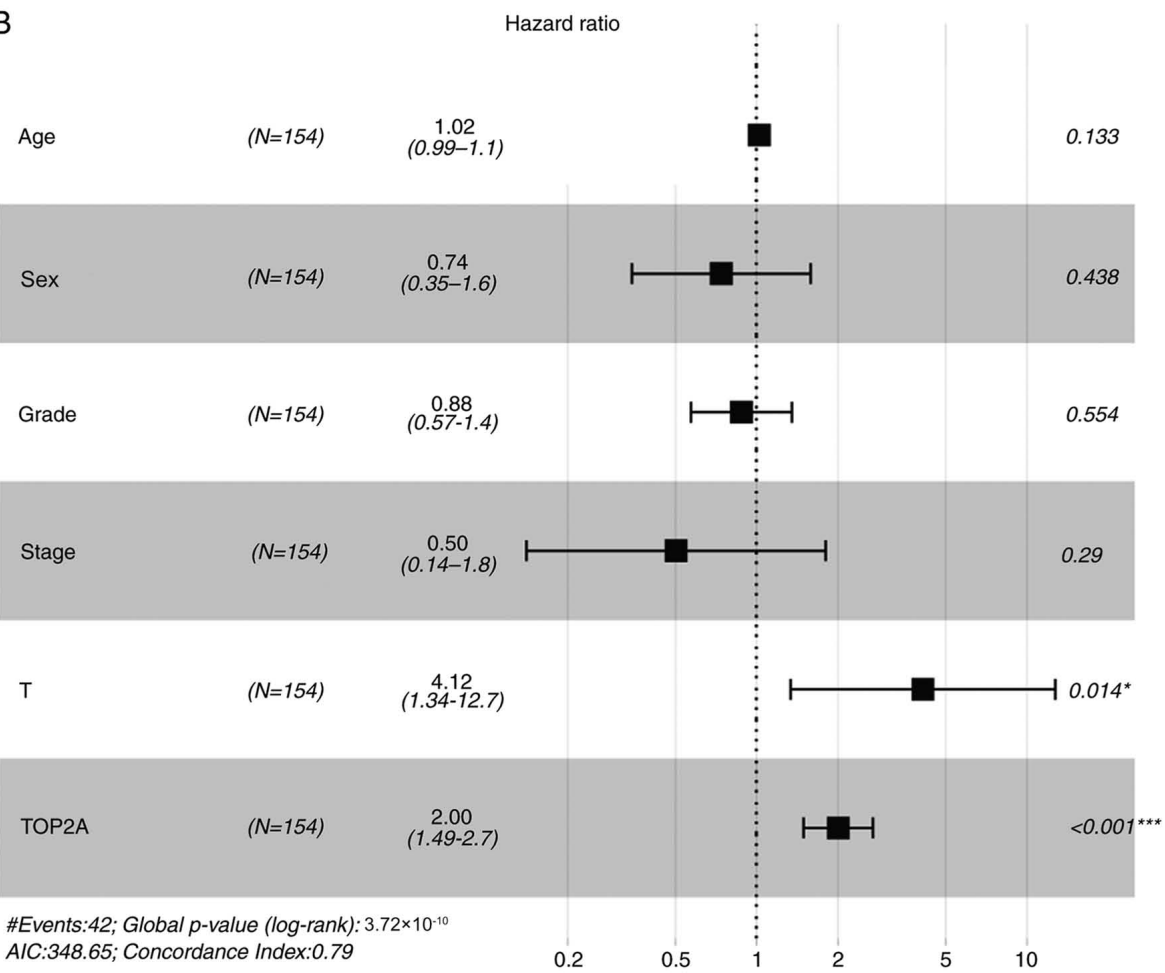

Figure 5. Multivariate analysis of prognostic factors in hepatocellular carcinoma. (A) All cases were fromTCGA cohort. (B) Asian cases in TCGA cohort. TOP2A, DNA topoisomerase Ii $\alpha$; stage, clinical stage; T, tumor size stage; TCGA, The Cancer Genome Atlas.

adenocarcinoma, bladder urothelial carcinoma, breast, prostate and colon cancer, indicates a poor prognosis (51-55), although this has not been previously reported in HCC. However, Panvichian et al (56) have demonstrated that the high expression of TOP2A in $\mathrm{HCC}$ is associated with the high expression of $\mathrm{Ki} 67$, and $\mathrm{Ki}-67$ expression has been found to correlate with tumor growth rate and poor prognosis in HCC (57), which indicates that TOP2A is a potential target for the treatment of HCC. In the present study, TOP2A was selected as a potential prognostic marker of HCC for further study. Analysis of the differential expression of TOP2A in 50 pairs of tumor and paracancerous tissue specimens in TCGA database revealed that the expression of TOP2A in tumor tissue was significantly higher compared with that in paracancerous tissue. In fresh 

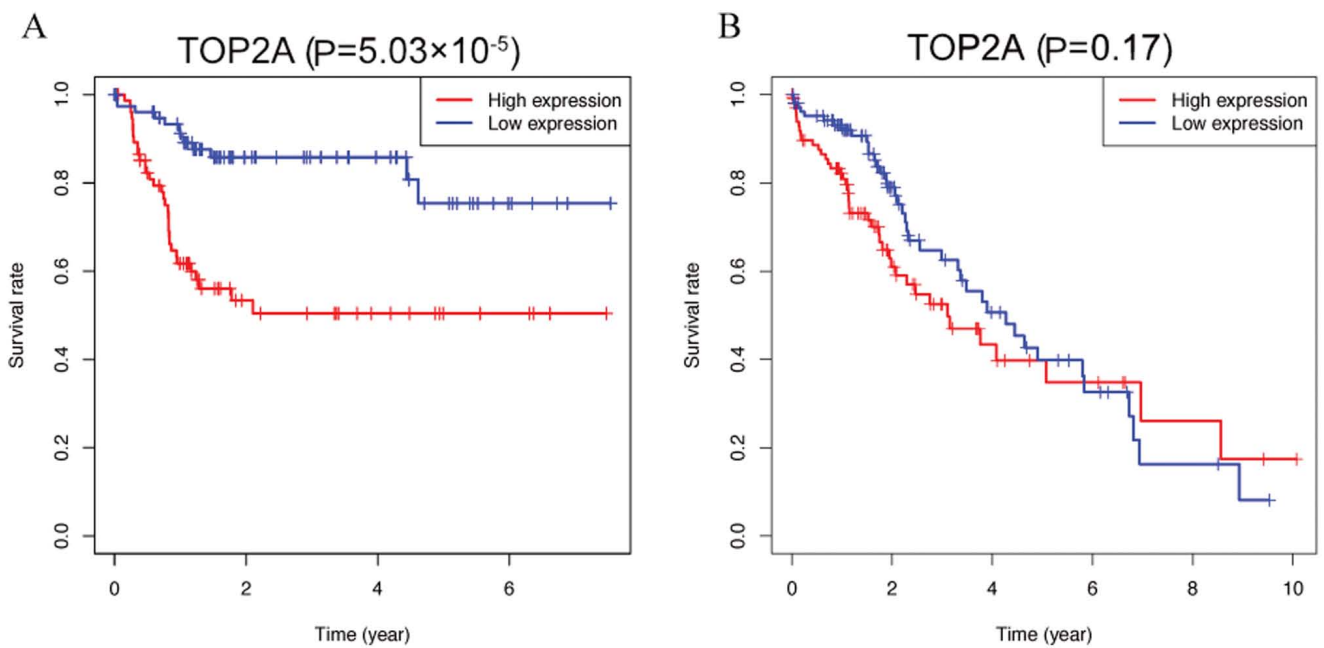

Figure 6. Overall survival in patients with hepatocellular carcinoma stratified into groups by TOP2A expression. (A) Overall survival in the Asian population in TCGA cohort. (B) Overall survival in the non-Asian population in TCGA cohort. TOP2A, DNA topoisomerase II $\alpha$; TCGA, The Cancer Genome Atlas.

clinical samples, the mRNA levels in HCC and paired samples confirmed that TOP2A was significantly highly expressed in tumor tissues, consistent with a previous study (56), suggesting that TOP2A may be associated with the occurrence and development of liver cancer. These results suggested that the high expression levels of TOP2A in HCC may be associated with tumor growth and metastasis, and that TOP2A may be used as a biomarker to predict tumor prognosis in clinical practice.

In the present study, a high $\mathrm{T}$ stage, poor differentiation degree and a high clinical stage were associated with high expression levels of TOP2A. In the univariate and multivariate analyses, high expression of TOP2A was identified as an independent predictor of HCC prognosis. Zhang et al (53) have reported that TOP2A promotes colon cancer progression by regulating ERK and AKT. The ERK and AKT pathways are two key signaling pathways involved in various tumor processes associated with the malignant proliferation of cancer $(58,59)$. Therefore, in HCC, TOP2A may also promote the proliferation of $\mathrm{HCC}$ by regulating these two pathways.

The results of the present study demonstrated that the cumulative survival rate of patients with high expression of TOP2A was higher compared with that of patients with low expression of TOP2A. Due to ethnic differences, the role of TOP2A expression in predicting tumor prognosis in different ethnic groups was also different (60). The association between high expression of TOP2A and poor prognosis was more significant in the Asian population compared with that the white population in the present study. The etiology of Asian HCC is mostly associated with viral hepatitis (37), which may be due to the effects of the hepatitis virus on DNA replication. Thus, the predictive effect of TOP $2 \mathrm{~A}$ on $\mathrm{HCC}$ may be different between the Asian and non-Asian populations.

However, there were certain limitations to the present study. First, the present study determined that high expression of TOP2A may lead to a poor prognosis of HCC using TCGA; while TCGA is a useful resource for obtaining research clues, this result needs to be verified in clinical samples, which will be performed in future studies. Second, since the present study was preliminary, the difference in the expression of TOP2A in 15 pairs of tumors and paracancerous tissues was only verified at the mRNA level, and these differences will be verified at the protein level in more cases in the future study. Third, in the survival analysis between the high and low TOP2A expression groups, a number of survival curves reversed in the late follow-up period. As Rocha-Singh (61) has reported, retrospective and observational studies present inherent potential moderate and strong bias, which may obscure, overestimate and even reverse the real effects. The appearance of this reversal may mean that beyond this time point, the value of TOP2A as a risk factor for poor prognosis of $\mathrm{HCC}$ began to decrease. On the other hand, cases in the TCGA database are followed up for $\leq 10$ years. With the extension of survival time, a number of patients may succumb to other diseases than HCC, which may lead to bias in the results at the later stage of follow-up. In addition, further studies will be required in the future to establish the prediction model, in vitro and in vivo functions, signal transduction pathways and molecular mechanisms that affect the expression of TOP2A in HCC.

In conclusion, the results of the present study demonstrated that high expression of TOP2A in HCC was associated with an advanced clinical stage, a low tumor differentiation grade and a high $\mathrm{T}$ stage. The expression of TOP2A may be an important biomarker for predicting the prognosis of HCC, especially in the Asian population.

\section{Acknowledgements}

Not applicable.

\section{Funding}

This work was supported by the Nantong Science and Technology Plan (grant no. MSZ18223), the Nantong Youth Medical Talent Fund (grant no. Youth 088) and the Youth Fund of Nantong Health Commission (grant no. WKZL2018045).

\section{Availability of data and materials}

The datasets used or analysed during the current study are available from the corresponding author on reasonable request. 


\section{Authors' contributions}

$\mathrm{ZC}$ and $\mathrm{HC}$ were responsible for the conceptualization of the study, drafting and revision of the manuscript. BS and $\mathrm{YZ}$ performed the experiments and analyzed the data. All the authors read and approved the final manuscript.

\section{Ethics approval and consent to participate}

The study protocol and acquisition of tissue specimens were approved by the Clinical Ethics Committee of The Affiliated Tumor Hospital of Nantong University (Nantong, China) (approval no. 2018-024) and all patients provided signed informed consent.

\section{Patient consent for publication}

Not applicable.

\section{Competing interests}

The authors declare that they have no competing interests.

\section{References}

1. WHO: Cancer today. https://gco.iarc.fr/today/home. Accessed June 242019.

2. Daher S, Massarwa M, Benson AA and Khoury T: Current and future treatment of hepatocellular carcinoma: An updated comprehensive review. J Clin Transl Hepatol 6: 69-78, 2018.

3. Park SJ, Jang JY, Jeong SW, Cho YK, Lee SH, Kim SG, Cha SW, Kim YS, Cho YD, Kim HS, et al: Usefulness of AFP, AFP-L3, and PIVKA-II, and their combinations in diagnosing hepatocellular carcinoma. Medicine (Baltimore) 96: e5811, 2017.

4. Sharma SA, Kowgier M, Hansen BE, Brouwer WP, Maan R, Wong D, Shah H, Khalili K, Yim C, Heathcote EJ, et al: Toronto HCC risk index: A validated scoring system to predict 10 -year risk of HCC in patients with cirrhosis. J Hepatol: Aug 24, 2017 (Epub ahead of print). doi: 10.1016/j.jhep.2017.07.033.

5. Shen J, He L, Li C, Wen T, Chen W, Lu C, Yan L, Li B and Yang J: Nomograms to predict the individual survival of patients with solitary hepatocellular carcinoma after hepatectomy. Gut Liver 11: 684-692, 2017.

6. Visone V, Vettone A, Serpe M, Valenti A, Perugino G, Rossi M and Ciaramella M: Chromatin structure and dynamics in hot environments: Architectural proteins and DNA topoisomerases of thermophilic archaea. Int J Mol Sci 15: 17162-17187, 2014.

7. Choi IY, Chung IK and Muller MT: Eukaryotic topoisomerase II cleavage is independent of duplex DNA conformation. Biochim Biophys Acta 1264: 209-214, 1995.

8. Austin CA, Lee KC, Swan RL, Khazeem MM, Manville CM, Cridland P, Treumann A, Porter A, Morris NJ and Cowell IG: TOP2B: The first thirty years. Int J Mol Sci 19: 2765, 2018.

9. Bush NG, Evans-Roberts K and Maxwell A: DNA topoisomerases. EcoSal Plus 6: 2015

10. Antoniou-Kourounioti M, Mimmack ML, Porter ACG and Farr CJ: The impact of the $\mathrm{C}$-terminal region on the interaction of topoisomerase II alpha with mitotic chromatin. Int J Mol Sci 20: 1238, 2019.

11. Demoulin B, Hermant M, Castrogiovanni C, Staudt C and Dumont P: Resveratrol induces DNA damage in colon cancer cells by poisoning topoisomerase II and activates the ATM kinase to trigger p53-dependent apoptosis. Toxicol In Vitro 29: $1156-1165,2015$

12. Sudan S and Rupasinghe HPV: Flavonoid-enriched apple fraction AF4 induces cell cycle arrest, DNA topoisomerase II inhibition, and apoptosis in human liver cancer HepG2 cells. Nutr Cancer 66: 1237-1246, 2014.
13. Kania EE, Carvajal-Moreno J, Hernandez VA, English A Papa JL, Shkolnikov N, Ozer HG, Yilmaz AS, Yalowich JC and Elton TS: hsa-miR-9-3p and hsa-miR-9-5p as post-transcriptional modulators of DNA topoisomerase II $\alpha$ in human leukemia K562 cells with acquired resistance to etoposide. Mol Pharmacol 97: $159-170,2020$

14. Kanagasabai R, Karmahapatra S, Kientz CA, Yu Y, Hernandez VA, Kania EE, Yalowich JC and Elton TS: The novel c-terminal truncated $90-\mathrm{kDa}$ isoform of topoisomerase II (TOP2 $\alpha / 90)$ is a determinant of etoposide resistance in K562 leukemia cells via heterodimerization with the TOP $2 \alpha / 170$ isoform. Mol Pharmacol 93: 515-525, 2018.

15. D Arcy $\mathrm{N}$ and Gabrielli B: Topoisomerase II inhibitors and poisons, and the influence of cell cycle checkpoints. Curr Med Chem 24: 1504-1519, 2017.

16. Wang JC: Cellular roles of DNA topoisomerases: A molecular perspective. Nat Rev Mol Cell Biol 3: 430-440, 2002.

17. Jain CK, Roychoudhury S and Majumder HK: Selective killing of G2 decatenation checkpoint defective colon cancer cells by catalytic topoisomerase II inhibitor. Biochim Biophys Acta 1853: 1195-1204, 2015.

18. Deng SP and Guo WL: Identifying key genes of liver cancer by networking of multiple data sets. IEEE/ACM Trans Comput Biol Bioinform 16: 792-800, 2019.

19. Yu Y, Ding S, Liang Y, Zheng Y, Li W, Yang L, Zheng X and Jiang J: Expression of ERCC1, TYMS, TUBB3, RRM1 and TOP2A in patients with esophageal squamous cell carcinoma: A hierarchical clustering analysis. Exp Ther Med 7: 1578-1582, 2014.

20. Xing C, Cai Z, Gong J, Zhou J, Xu J and Guo F: Identification of potential biomarkers involved in gastric cancer through integrated analysis of non-coding RNA associated competing endogenous RNAs network. Clin Lab 64: 1661-1669, 2018.

21. Liu LM, Xiong DD, Lin P, Yang H, Dang YW and Chen G: DNA topoisomerase 1 and $2 \mathrm{~A}$ function as oncogenes in liver cancer and may be direct targets of nitidine chloride. Int J Oncol 53: 1897-1912, 2018.

22. Eltohamy MI, Badawy OM, El kinaai N, Loay I, Nassar HR, Allam RM and Ali Sakr M: Topoisomerase II $\alpha$ gene alteration in triple negative breast cancer and its predictive role for anthracycline-based chemotherapy (Egyptian NCI Patients). Asian Pac J Cancer Prev 19: 3581-3589, 2018.

23. Wittmann S, Wunder C, Zirn B, Furtwängler R, Wegert J, Graf N and Gessler M: New prognostic markers revealed by evaluation of genes correlated with clinical parameters in wilms tumors. Genes Chromosomes Cancer 47: 386-395, 2008.

24. Xu XL, Zheng WH, Fu ZX, Li ZP, Xie HX, Li XX, Jiang LH, Wang Y, Zhu SM and Mao WM: Topo2A as a prognostic biomarker for patients with resectable esophageal squamous cell carcinomas. Med Oncol 32: 396, 2015.

25. de Resende MF, Vieira S, Chinen LT, Chiappelli F, da Fonseca FP, Guimarães GC, Soares FA, Neves I, Pagotty S, Pellionisz PA, et al: Prognostication of prostate cancer based on TOP2A protein and gene assessment: TOP2A in prostate cancer. J Transl Med 11: 36, 2013.

26. Sahin S, Gönül II, Cakır A, Seçkin S and Uluoğlu O: Clinicopathological significance of the proliferation markers Ki67, RacGAP1, and topoisomerase 2 alpha in breast cancer. Int J Surg Pathol 24: 607-613, 2016.

27. Pei YF, Yin XM and Liu XQ: TOP2A induces malignant character of pancreatic cancer through activating $\beta$-catenin signaling pathway. Biochim Biophys Acta Mol Basis Dis 1864: 197-207, 2018.

28. Labbe DP, Sweeney CJ, Brown M, Galbo P, Rosario S, Wadosky KM, Ku SY, Sjöström M, Alshalalfa M, Erho N, et al: TOP2A and EZH2 provide early detection of an aggressive prostate cancer subgroup. Clin Cancer Res 23: 7072-7083, 2017.

29. Villman K, Sjöström J, Heikkilä R, Hultborn R, Malmström P, Bengtsson NO, Söderberg M, Saksela E and Blomqvist C: TOP2A and HER2 gene amplification as predictors of response to anthracycline treatment in breast cancer. Acta Oncol 45: 590-596, 2006.

30. Ito F, Furukawa $\mathrm{N}$ and Nakai T: Evaluation of TOP2A as a predictive marker for endometrial cancer with taxane-containing adjuvant chemotherapy. Int J Gynecol Cancer 26: 325-330, 2016.

31. Roca E, Berruti A, Sbiera S, Rapa I, Oneda E, Sperone P, Ronchi CL, Ferrari L, Grisanti S, Germano A, et al: Topoisomerase $2 \alpha$ and thymidylate synthase expression in adrenocortical cancer. Endocr Relat Cancer 24: 319-327, 2017. 
32. Cai HY, Zhu XH, Qian F, Shao B, Zhou Y, Zhang Y and Chen Z: High expression of TOP2A gene predicted poor prognosis of hepatocellular carcinoma after radical hepatectomy. Transl Cancer Res 9: 983-992, 2020.

33. Livak KJ and Schmittgen TD: Analysis of relative gene expression data using real-time quantitative PCR and the 2(-Delta Delta C(T)) method. Methods 25: 402-408, 2001.

34. R Core Team (2014). R: A language and environment for statistical computing. R Foundation for Statistical Computing, Vienna, Austria. URL http://www.R-project.org/

35. Ritchie ME, Phipson B, Wu D, Hu Y, Law CW, Shi W and Smyth GK: limma powers differential expression analyses for RNA-sequencing and microarray studies. Nucleic Acids Res 43: e47, 2015.

36. Ecklund A: beeswarm: The Bee Swarm Plot, an Alternative to Stripchart, https://CRAN.R-project.org/package=beeswarm.

37. Kulik L and El-Serag HB: Epidemiology and management of hepatocellular carcinoma. Gastroenterology 156: 477-491 e471, 2019.

38. Yau T, Hsu C, Kim TY, Choo SP, Kang YK, Hou MM, Numata K, Yeo W, Chopra A, Ikeda M, et al: Nivolumab in advanced hepatocellular carcinoma: Sorafenib-experienced Asian cohort analysis. J Hepatol 71: 543-552, 2019.

39. Di Bisceglie AM and Hoofnagle JH: Elevations in serum alpha-fetoprotein levels in patients with chronic hepatitis B. Cancer 64: 2117-2120, 1989.

40. Agopian VG, Harlander-Locke MP, Markovic D, Zarrinpar A Kaldas FM, Cheng EY, Yersiz H, Farmer DG, Hiatt JR and Busuttil RW: Evaluation of patients with hepatocellular carcinomas that do not produce $\alpha$-fetoprotein. JAMA Surg 152: 55-64, 2017.

41. Luo P, Wu S, Yu Y, Ming X, Li S, Zuo X and Tu J: Current status and perspective biomarkers in AFP negative HCC: Towards screening for and diagnosing hepatocellular carcinoma at an earlier stage. Pathol Oncol Res 26: 599-603, 2020.

42. European Association For The Study Of The Liver; European Organisation For Research and Treatment Of Cancer: EASL-EORTC clinical practice guidelines: Management of hepatocellular carcinoma. J Hepatol 56: 908-943, 2012.

43. Yan L, Xu F and Dai C: Overexpression of COL24A1 in hepatocellular carcinoma predicts poor prognosis: A study based on multiple databases, clinical samples and cell lines. Onco Targets Ther 13: 2819-2832, 2020.

44. Luo YD, Yu HQ, Liu XY, Huang D, Dai HS, Fang L, Zhang YJ, Lai JJ, Jiang Y, Shuai L, et al: Prognostic and predicted significance of Ubqln2 in patients with hepatocellular carcinoma. Cancer Med 9: 4083-4094, 2020.

45. Chen L, Liu D, Yi X, Qi L, Tian X, Sun B, Dong Q, Han Z, Li Q, Song T, et al: The novel miR-1269b-regulated protein SVEP1 induces hepatocellular carcinoma proliferation and metastasis likely through the PI3K/Akt pathway. Cell Death Dis 11: 320, 2020 .

46. Chen J, Ding C, Chen Y, Hu W, Lu Y, Wu W, Zhang Y, Yang B, Wu H, Peng C, et al: ACSL4 promotes hepatocellular carcinoma progression via c-Myc stability mediated by ERK/FBW7/c-Myc axis. Oncogenesis 9: 42, 2020.

47. Zhao X, Chen Y, Mao Q, Jiang X, Jiang W, Chen J, Xu W, Zhong $\mathrm{L}$ and Sun X: Overexpression of YTHDF1 is associated with poor prognosis in patients with hepatocellular carcinoma. Cancer Biomark 21: 859-868, 2018.
48. Li F, Bai L, Li S, Chen Y, Xue X and Yu Z: Construction and evaluation of a prognosis lncRNA model for hepatocellular carcinoma. J Cell Biochem: Apr 29, 2020 (Epub ahead of print). doi: https://doi.org/10.1002/jcb.29608.

49. Kou F, Sun H, Wu L, Li B, Zhang B, Wang X and Yang L: TOP2A promotes lung adenocarcinoma cells' malignant progression and predicts poor prognosis in lung adenocarcinoma. J Cancer 11: 2496-2508, 2020

50. Liu T, Zhang H, Yi S, Gu L and Zhou M: Mutual regulation of MDM4 and TOP2A in cancer cell proliferation. Mol Oncol 13: 1047-1058, 2019.

51. Zeng S, Liu A, Dai L, Yu X, Zhang Z, Xiong Q, Yang J, Liu F, $\mathrm{Xu}$ J, Xue Y, et al: Prognostic value of TOP2A in bladder urothelial carcinoma and potential molecular mechanisms. BMC Cancer 19: 604, 2019.

52. Badawy OM and Loay I: FISH analysis of TOP2A and HER-2 aberrations in female breast carcinoma on archived material: Egyptian NCI experience. Appl Immunohistochem Mol Morphol 27: 216-222, 2019.

53. Zhang R, Xu J, Zhao J and Bai JH: Proliferation and invasion of colon cancer cells are suppressed by knockdown of TOP2A. J Cell Biochem 119: 7256-7263, 2018.

54. Xiong Y, Yuan L, Chen L, Zhu Y, Zhang S, Liu X, Xiao Y and Wang X: Identifying a novel biomarker TOP2A of clear cell renal cell carcinoma (ccRCC) associated with smoking by co-expression network analysis. J Cancer 9: 3912-3922, 2018.

55. Nelson WG, Haffner MC and Yegnasubramanian S: The structure of the nucleus in normal and neoplastic prostate cells: Untangling the role of type 2 DNA topoisomerases. Am J Clin Exp Urol 6: 107-113, 2018.

56. Panvichian R, Tantiwetrueangdet A, Angkathunyakul N and Leelaudomlipi S: TOP2A amplification and overexpression in hepatocellular carcinoma tissues. Biomed Res Int 2015: 381602 , 2015.

57. Ng IO, Na J, Lai EC, Fan ST and Ng M: Ki-67 antigen expression in hepatocellular carcinoma using monoclonal antibody MIB1. A comparison with proliferating cell nuclear antigen. Am J Clin Pathol 104: 313-318, 1995.

58. Steelman LS, Chappell WH, Abrams SL, Kempf RC, Long J, Laidler P, Mijatovic S, Maksimovic-Ivanic D, Stivala F, Mazzarino MC, et al: Roles of the Raf/MEK/ERK and PI3K/PTEN/Akt/mTOR pathways in controlling growth and sensitivity to therapy-implications for cancer and aging. Aging (Albany NY) 3: 192-222, 2011.

59. Zhao J, Ou B, Han D, Wang P, Zong Y, Zhu C, Liu D, Zheng M, Sun J, Feng H and Lu A: Tumor-derived CXCL5 promotes human colorectal cancer metastasis through activation of the ERK/Elk-1/Snail and AKT/GSK3 $/ / \beta$-catenin pathways. Mol Cancer 16: 70, 2017.

60. Echevarria MI, Awasthi S, Cheng CH, Berglund AE, Rounbehler RJ, Gerke TA, Takhar M, Davicioni E, Klein EA, Freedland SJ, et al: African American specific gene panel predictive of poor prostate cancer outcome. J Urol 202: 247-255, 2019.

61. Rocha-Singh KJ: Retrospective real-world studies of paclitaxel and mortality: Defining the many faces of bias. JACC Cardiovase Interv: May 14, 2020 (Epub ahead of print). doi: 10.1016/j. jcin.2020.05.006.

(i) $(-)$ This work is licensed under a Creative Commons Attribution-NonCommercial-NoDerivatives 4.0 International (CC BY-NC-ND 4.0) License. 